\title{
IMAGE HALFTONING USING OPTIMIZED DOT DIFFUSION
}

\author{
Murat Meşe and P.P. Vaidyanathan \\ Department of Electrical Engineering 136-93, \\ California Institute of Technology,Pasadena, CA 91125 USA, \\ Phone (626) 395-4681 Fax: (626) 795-8649 \\ E-mail: mese@systems.caltech.edu, ppvnath@sys.caltech.edu
}

\begin{abstract}
The dot diffusion method for digital halftoning has the advantage of parallelism unlike the error diffusion method. However, image quality offered by error diffusion is still regarded as superior to other known methods. In this paper we show how the dot diffusion method can be improved by optimization of the so-called class matrix. By taking the human visual characteristics into account we show that such optimization consistently results in images comparable to error diffusion, without sacrificing the parallelism.
\end{abstract}

\section{INTRODUCTION}

Digital halftoning is the rendition of continuous-tone pictures on displays that are capable of producing only two levels. There are many good methods for digital halftoning: ordered dither [1], error diffusion [2], neural-net based methods [8], and more recently direct binary search (DBS) [7]. Ordered dithering is a thresholding of the continuous-tone image with a spatially periodic screen [1] . In error diffusion [2], the error is 'diffused' to the unprocessed neighbor points.

Ordered dithering is a parallel method, requiring only pointwise comparisons. But the resulting halftones suffer from periodic patterns. On the other hand error diffused halftones do not suffer from periodicity and offer blue noise characteristic [3] which is found to be desirable. The main drawback is that error diffusion is inherently serial, i.e., to get the halftoned value of the last pixel, all of the remaining points should be processed. Also there occur worm-like patterns in near mid-gray regions and resulting halftones have ghosting problem [6]. Mitsa and Parker have optimized ordered dither matrix [4] for large size like $256 \times 256$ to get the blue noise effect. This is a compromise between parallelism and image quality.

The dot diffusion method for halftoning, introduced by Knuth [6] is an attractive method which attempts to retain the good features of error diffusion while offering substantial parallelism. However, surprisingly, not much work has been done on optimization of the so-called class matrix. In this work we will show that the class matrix (see below) can further be optimized by taking into account the properties of human visual system (HVS). The resulting halftones will then be of the same quality as for error diffusion. Since dot diffusion also offers increased parallelism, it now appears to be an attractive alternative to error diffusion.

\footnotetext{
${ }^{1}$ This work was supported by Office of Naval Research Grant N00014-93-1-0231.
}

\section{REVIEW OF DOT DIFFUSION}

The dot diffusion method for halftoning has only one design parameter, called class matrix C. It determines the order in which the pixels are halftoned. Thus, the pixel positions $\left(n_{1}, n_{2}\right)$ of an image are divided into $I J$ classes according to $\left(n_{1} \bmod I, n_{2} \bmod J\right)$ where $I$ and $J$ are constant integers. Table 1 shows an example of the class matrix for $I=J=8$, used by Knuth. There are 64 class numbers. Let $x\left(n_{1}, n_{2}\right)$ be the contone image with pixel values in the normalized range $[0,1]$. Starting from class $k=1$, we process the pixels for increasing values of $k$. For a fixed $k$, we take all pixel locations $\left(n_{1}, n_{2}\right)$ belonging to class $k$ and define the halftone pixels to be

$$
x_{h}\left(n_{1}, n_{2}\right)= \begin{cases}1 & \text { if } x\left(n_{1}, n_{2}\right) \geq 0.5 \\ 0 & \text { if } x\left(n_{1}, n_{2}\right)<0.5\end{cases}
$$

We also define the error $e\left(n_{1}, n_{2}\right)=x\left(n_{1}, n_{2}\right)-x_{h}\left(n_{1}, n_{2}\right)$. We then look at the eight neighbors of $\left(n_{1}, n_{2}\right)$ and replace the contone pixel with an adjusted version for those neighbors which have a higher class number (i.e., those neighbors that have not been halftoned yet). To be specific, neighbors with higher class numbers are replaced with

$$
\begin{array}{rcc}
x(i, j)+2 e\left(n_{1}, n_{2}\right) / w & \text { (for orthogonal neighbors) } & (1(a)) \\
x(i, j)+e\left(n_{1}, n_{2}\right) / w & \text { (for diagonal neighbors) } & (1(b))
\end{array}
$$

where $w$ is such that the sum of errors added to all the neighbors is exactly $e\left(n_{1}, n_{2}\right)$. The extra factor of two for orthogonal neighbors (i.e., vertically and horizontally adjacent neighbors) is because vertically or horizontally oriented error patterns are more perceptible than diagonal patterns.

The contone pixels $x\left(n_{1}, n_{2}\right)$ which have the next class number $k+1$ are then similarly processed. The pixel values $x\left(n_{1}, n_{2}\right)$ are of course not the original contone values but the adjusted values according to earlier diffusion steps (1). When the algorithm terminates, the signal $x_{h}\left(n_{1}, n_{2}\right)$ is the desired halftone.

This diffusion process is illustrated in Fig. 1. The numbers in the matrix are elements of a class matrix and the integers in the bubbles are relative weights of diffusion coefficients. The neighbors of 33 with higher class number are those labeled as $58,45,42,40,63,47$. The error created at 33 is divided by the sum of relative weights of diffusion coefficients, which is $2+1+2+1+2+1=9$ in this case. The result of the division, $\epsilon$, is the error to be diffused to diagonal 


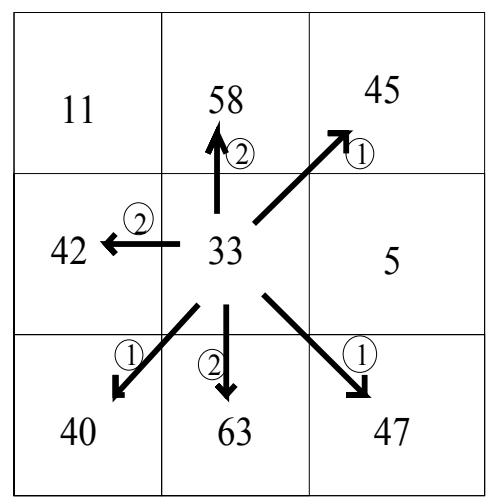

Figure 1: Error Diffusion from a point to the neighbor points

neighbors, and $2 \epsilon$ is diffused to orthogonal neighbors. In 64 steps the algorithm will complete the halftoning process.

Usually an image is enhanced [6] before dot diffusion is applied. For this the continuous image pixels $C(i, j)$ are replaced by $C^{\prime}(i, j)=\frac{C(i, j)-\alpha \bar{C}(i, j)}{1-\alpha}$ where $\bar{C}(i, j)=$ $\frac{\sum_{i-1}^{i+1} \sum_{j-1}^{j+1} C(i, j)}{9}$. If $\alpha=0.9$ then

$$
C^{\prime}(i, j)=8 C(i, j)+C(i, j)-\sum_{0<(u-i)^{2}+(v-j)^{2}<3} C(u, v)
$$

This algorithm is completely parallel requiring 9 additions per pixel, and no multiplications.

\section{OPTIMIZATION OF CLASS MATRIX}

Knuth introduced the notion of barons and near barons in the selection of his class matrix. A baron has only low-class neighbors, and a near-baron has one high class neighbor. The quantization error at a baron cannot be distributed to neighbors, and the error at a near baron can be distributed to only one neighbor. Knuth's idea was that the number of Barons and near barons should therefore be minimized. He exhibited a class matrix with two barons and two near barons (Table 1). The quality of the resulting halftones are still inferior to error diffusion because of periodic patterns similar to ordered dither methods (see Fig. 6). Knuth has produced a class matrix with one baron and near baron, but unfortunately these line up to produced objectionable visual artifacts. In our experience, the baron near baron criterion does not appear to be the right choice for optimization. To explain this, define a $k$-baron to be a position which has $k$ high-level neighbors. Thus $k=0$ corresponds to a baron, $k=1$ to a near baron, and $k=8$ to an antibaron. We have produced a class matrix which minimizes the number of $k$ barons sequentially for $0 \leq k \leq 8$. The resulting halftone quality was found in most cases to be slightly worse than Knuth's original results, leading us to conclude that baron minimization is not the right approach. In Sec. 3.1 we introduce a different optimization criterion based on the HVS, and show that the image quality is significantly improved, though the class matrix does not minimize barons.

\subsection{Objective Function Based on Blue Noise}

It has been observed in the past that the error in a good halftone should have the blue noise property [3]. This means that the noise energy should mostly be in the high frequency region where it is known to be less perceptible. We will show how to incorporate blue noise characteristics into the class matrix optimization.

Imagine that we have a constant gray image $x\left(n_{1}, n_{2}\right)=g$ where $0 \leq g \leq 1$. Let $x_{h}\left(n_{1}, n_{2}\right)$ denote the halftoned version. Since the halftone is supposed to create the perception of the gray level, the average number of dark pixels should be equal to the original gray level. Typically, therefore, the dark pixels are spatially distributed with a certain average frequency $f_{g}$ called the principal frequency, which increases with gray level $g$. The preference for blue noise [3] (high frequency white noise) in halftoning arises because noise energy at a significantly higher spatial frequency than $f_{g}$ is not perceivable. Thus, we can optimize a halftoning method for a particular gray level $g$ by forcing the noise spectrum to be concentrated above $f_{g}$.

This does not, however, imply optimality at other gray levels. Interestingly however, if the gray level $g$ during the optimization phase is chosen carefully, the resulting halftones for arbitrary natural images are excellent. For example we optimized the class matrix in the dot diffusion method for the gray level $g=0.0625$ and obtained very good halftones for natural images as we demonstrate in Sec. 4 .

Calculating the noise spectrum. In order to implement the optimization, we first need to compute the noise spectrum. The halftone pattern $x_{h}\left(n_{1}, n_{2}\right)$ for the gray level $x\left(n_{1}, n_{2}\right)=$ $g$ has the error $e\left(n_{1}, n_{2}\right)=g-x_{h}\left(n_{1}, n_{2}\right)$, which is an $N \times N$ image. Imagine that this is divided into $L \times L$ blocks so there are $B=(N / L)^{2}$ blocks. (In our experiment $N=256, L=$ $64, B=16$.) Let $E_{m}\left(k_{1}, k_{2}\right)$ be the $L \times L$ DFT of the the $m$ th block of $e\left(n_{1}, n_{2}\right)$. We define the average noise spectrum as

$$
P\left(k_{1}, k_{2}\right)=\frac{1}{B} \sum_{m=0}^{B-1}\left|E_{m}\left(k_{1}, k_{2}\right)\right|^{2}
$$

From this we compute the so-called radially averaged power spectrum $P_{r}\left(k_{r}\right)$ where $k_{r}$ is a scalar called the radial frequency. Since $\left|k_{1}\right|$ and $\left|k_{2}\right|$ range from 0 to $L / 2, k_{r}$ ranges from 0 to $L / \sqrt{2}$. We take specific integer values for $k_{r}$ and calculate $P_{r}\left(k_{r}\right)$ as follows. For each chosen $k_{r}$ define an annulus $\mathcal{A}\left(k_{r}\right)$ in the $\left(k_{1}, k_{2}\right)$ plane by the equation

$$
\left|\sqrt{k_{1}^{2}+k_{2}^{2}}-k_{r}\right|<\Delta / 2
$$

The quantity $\Delta$, which determines the width of the annulus is chosen as unity in our work. With $N\left(k_{r}\right)$ denoting the number of elements in $\mathcal{A}\left(k_{r}\right)$, the radially averaged power spectrum of the error for gray level $g$ is then

$$
P_{r}\left(k_{r}\right)=\frac{1}{N\left(k_{r}\right)} \sum_{k_{1}, k_{2} \in \mathcal{A}\left(k_{r}\right)} P\left(k_{1}, k_{2}\right)
$$

The class matrix in the dot diffusion method should be optimized such that this radial spectrum is appropriately shaped for a well-chosen fixed gray level $g$. In terms of the radial frequency variable $k_{r}$, the principal frequency for the halftone of gray level $g$ is given by

$$
f_{g}=k_{\max } \sqrt{g}
$$


where $k_{\max }$ is the maximum value of $k_{r}$. In fact, for $g>$ 0.5 , since black pixels are more in number, the halftone is perceived as a distribution of white dots and we have to take [3] $f_{g}=k_{\max } \sqrt{1-g}$.
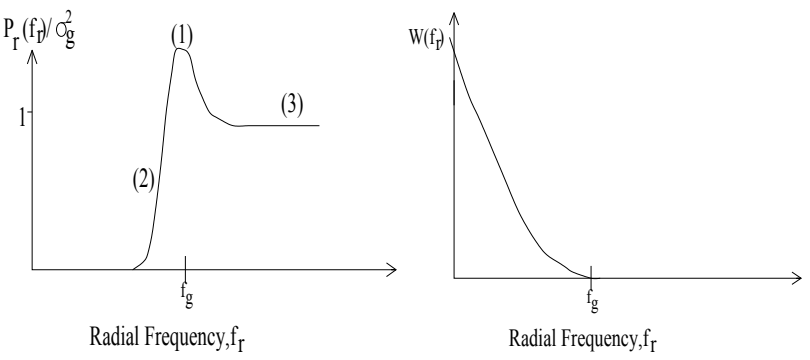

Figure 2: A Typical Desired Radial Spectrum Characteristics

Figure 3: The Weight Function used in the Optimization

The aim of the optimization is to shape $P_{r}\left(k_{r}\right)$ by choice of the class matrix $\mathbf{C}$ so that most of its energy is moved to the region $k_{r}>f_{g}$ (as demonstrated in Fig. 2). We therefore define the cost function

$$
\Phi(\mathbf{C}, g)=\int_{0}^{f_{g}} P_{r}\left(k_{r}\right) w\left(k_{r}\right) d k_{r}
$$

The idea is to choose the weighting function $w\left(k_{r}\right)$ such that upon minimization of the above function, $P_{r}\left(k_{r}\right)$ has a low frequency cutoff at principal frequency $f_{g}$, sharp transition region, and a flat high frequency region. The weight function was chosen to be $w\left(k_{r}\right)=c\left(k_{r}-f_{g}\right)^{2}$ for $0 \leq k_{r} \leq f_{g}$ and zero outside. The constant $c$ was chosen as unity. In the optimization the integral was replaced with a discrete sum. The choice of the class matrix that minimizes this sum was performed using the pairwise exchange algorithm [5] described below:

1) Randomly order the numbers in the class matrix.

2) List all possible exchanges of class numbers.

3) If an exchange does not reduce cost, restore the pair to original positions and proceed to the next pair.

4) If an exchange does reduce cost, keep it and restart the enumeration from the beginning.

5) Stop searching if no further exchanges reduce cost.

6) Repeat the above steps a fixed number of times and keep the best of the class matrix.

Choice of gray level. Since the algorithm can be applied only to a given gray level, the gray level should be chosen wisely, in order to get good halftones for other gray levels also. In our experience if we perform this optimization for a fixed small gray level (e.g., $g=0.0625,0.1$ etc.), we get good halftones for natural images also. Class matrices obtained from optimization with a very small gray level will not work, because there is not much error to diffuse to other points during the dot diffusion process. Mid gray levels are not suitable, first because there are huge diffusions between points, and second, even unoptimized algorithms yield perceptually pleasing halftones for mid gray anyway. The actual gray level used in the optimization is $g=0.0625$, and it is found experimentally. The class matrix obtained is shown in Table 2. Notice that the optimal class matrix has several barons and near barons.

\section{EXPERIMENTAL RESULTS}

Fig. 4 shows the $512 \times 512$ continuous tone peppers image. The halftone generated by using Knuth's class matrix is shown in Fig 6, and the halftone image generated by the optimized class matrix is shown in Fig 7 . It is clear that the new method is superior to unoptimized dot diffusion method. In fact, the new method offers a quality indistinguishable from FS error diffusion method (Fig. 5). Notice that the artificial periodic patterns in Fig. 6 are absent in Fig. 5 and in the new method Fig. 7.

\section{CONCLUSION}

Even though dot diffusion offers more parallelism than error diffusion, it has not received much attention. This is partly because the noise characteristics of error diffusion method are generally regarded as superior. In this paper we have shown that by optimizing the class matrix for blue noise at a fixed gray level, the results of dot diffusion can be made at least as pleasing as that of error diffusion. The algorithm terminates in at most 64 steps compared to $N^{2}$ steps needed for error diffusion. Moreover, since the optimized class matrix has eight barons and eight antibarons (Sec. 3.1), the algorithm can in fact be terminated in about 50 steps. The conclusion is that Knuth's dot diffusion method with a carefully optimized class matrix is very promising; the image quality is comparable to error diffusion, and the implementation offers more parallelism than error diffusion.

\section{References}

[1] B. E. Bayer, "An Optimum Method for Two Level Rendition of Continuous Tone Pictures, " in Conf. Rec. IEEE ICC, 1973, pp 26-11-26-15.

[2] R. W. Floyd and L. Steinberg, "An Adaptive Algorithm for Spatial Greyscale, "Proc. SID, vol. 17, no.2m pp. 75-77, 1976

[3] R. A. Ulichney, "Dithering with Blue Noise, "Proceedings of IEEE, vol. 76, no. 1, pp.56-79, January 1988.

[4] T. Mitsa and K. J. Parker, "Digital Halftoning Technique Using a Blue Noise Mask, "J. Opt. Soc. Am. A, Vol. 9, No. 11, pp. 1920-1929, November 1992.

[5] J.P. Allebach and R.N. Stradling, "Computer-aided Design of Dither Signals for Binary Display of Images, " Applied Optics, Vol. 18, No. 15, pp. 2708-2713, August 1979.

[6] D. E. Knuth, "Digital Halftones by Dot Diffusion, ", ACM Transactions on Graphics, vol. 6, no. 4, pp 245273, October 1987.

[7] M. A. Seldowitz, J. P. Allebach, and D. E. Sweeney, " Synthesis of digital holograms by direct binary search, " Appl. Opt Vol. 26, pp. 2788-2798, 1987.

[8] Dimitris Anastassiou, "Neural net based digital halftoning of images, "ISCAS, Vol. 1, pp. 507-510, 1988. 
Table 1: Class Matrix C used in Knuth's Method

\begin{tabular}{|l|l|l|l|l|l|l|l|}
\hline 35 & 49 & 41 & 33 & 30 & 16 & 24 & 32 \\
\hline 43 & 59 & 57 & 54 & 22 & 6 & 8 & 11 \\
\hline 51 & 63 & 62 & 46 & 14 & 2 & 3 & 19 \\
\hline 39 & 47 & 55 & 38 & 26 & 18 & 10 & 27 \\
\hline 29 & 15 & 23 & 31 & 36 & 50 & 42 & 34 \\
\hline 21 & 5 & 7 & 12 & 44 & 60 & 58 & 53 \\
\hline 13 & 1 & 4 & 20 & 52 & 64 & 61 & 45 \\
\hline 25 & 17 & 9 & 28 & 40 & 48 & 56 & 37 \\
\hline
\end{tabular}

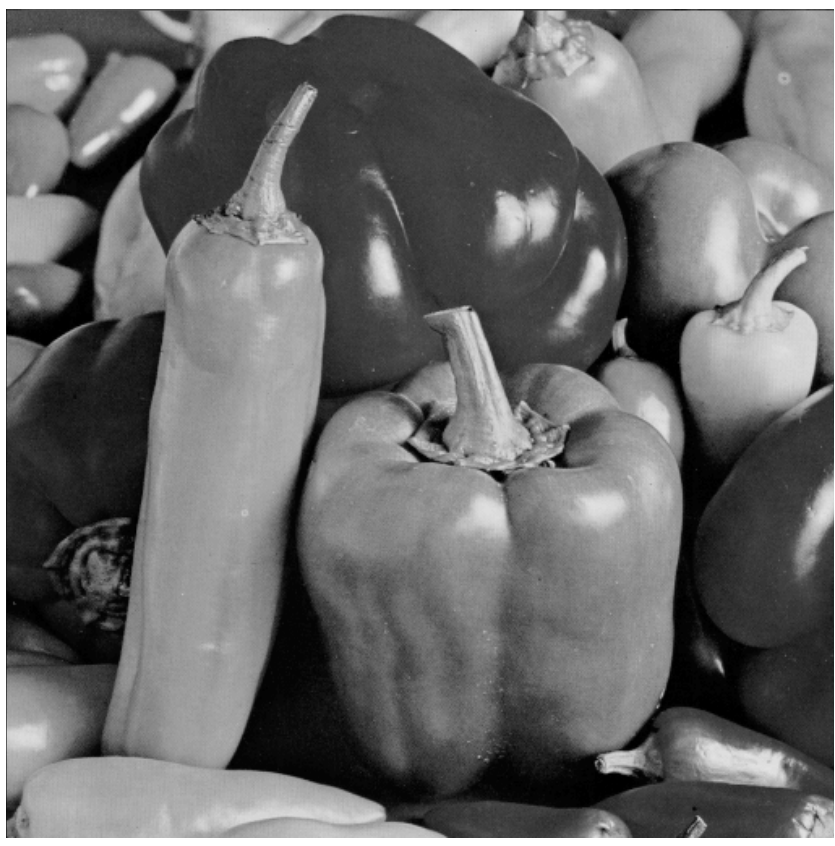

Figure 4: Original Image, Peppers

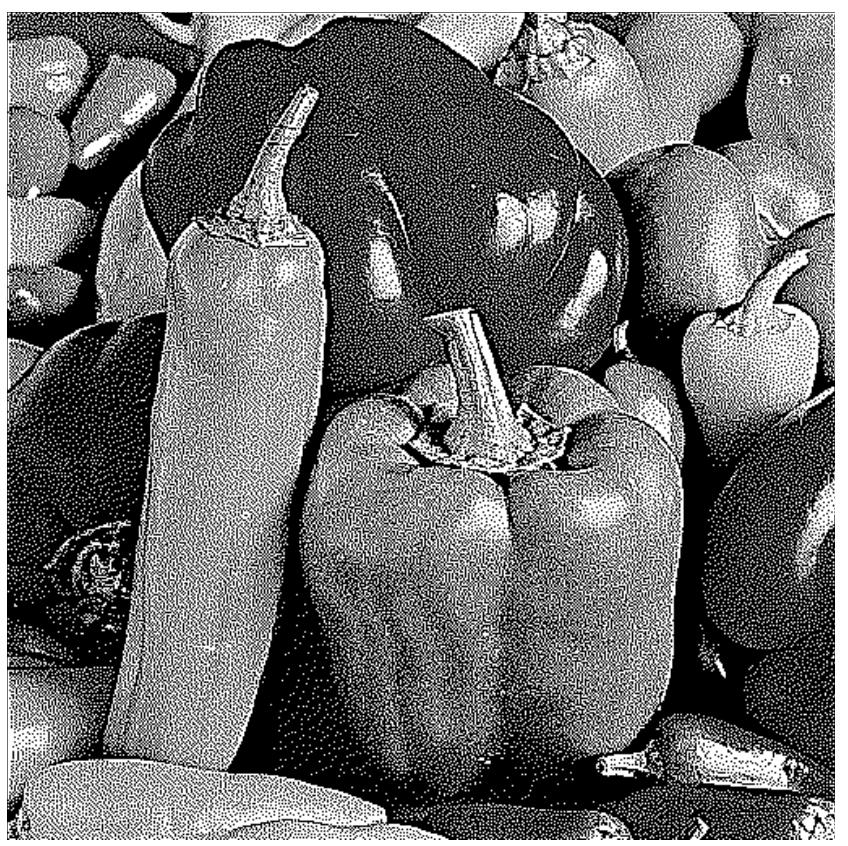

Figure 5: FS Error Diffusion
Table 2: Class Matrix $\mathbf{C}$ for the new method

\begin{tabular}{|l|l|l|l|l|l|l|l|}
\hline 59 & 12 & 46 & 60 & 28 & 14 & 32 & 3 \\
\hline 21 & 25 & 44 & 11 & 58 & 45 & 43 & 30 \\
\hline 24 & 20 & 13 & 42 & 33 & 5 & 54 & 8 \\
\hline 64 & 52 & 55 & 40 & 63 & 47 & 7 & 18 \\
\hline 35 & 57 & 9 & 15 & 50 & 48 & 4 & 36 \\
\hline 41 & 17 & 6 & 61 & 22 & 49 & 62 & 34 \\
\hline 2 & 53 & 19 & 56 & 39 & 23 & 26 & 51 \\
\hline 16 & 37 & 1 & 31 & 29 & 27 & 38 & 10 \\
\hline
\end{tabular}

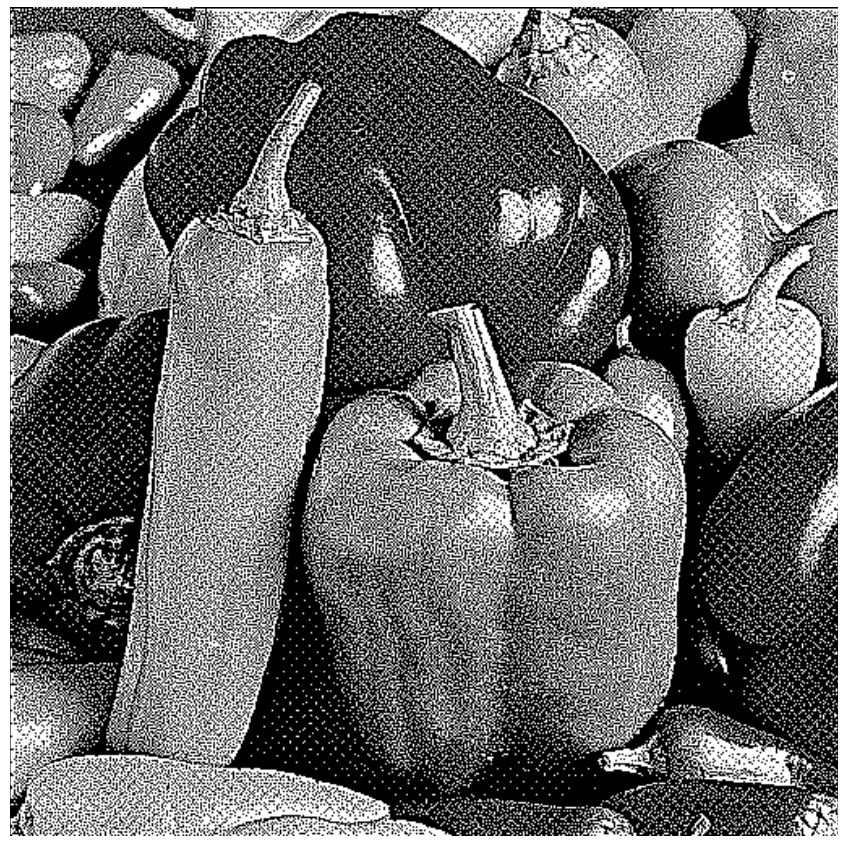

Figure 6: Dot Diffusion with Knuth's Class Matrix

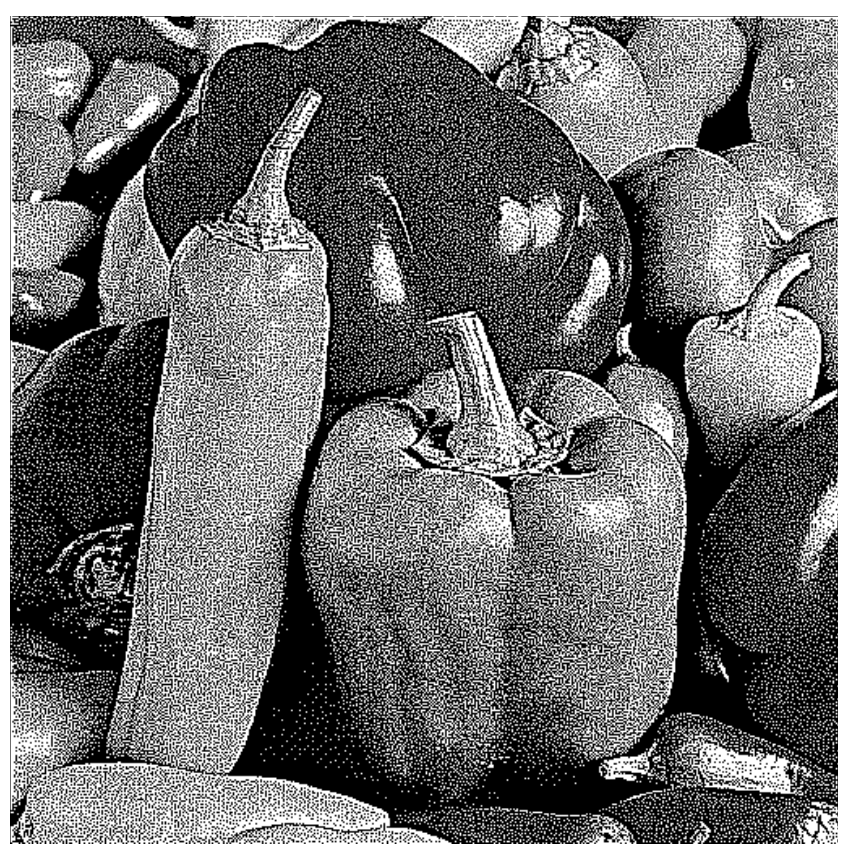

Figure 7: Dot Diffusion with the new Class Matrix 